Article

\title{
On the Real Homotopy Type of Generalized Complex Nilmanifolds
}

\author{
Adela Latorre ${ }^{1, *(1)}$, Luis Ugarte ${ }^{2}\left(\mathbb{C}\right.$ and Raquel Villacampa ${ }^{3}$ \\ 1 Departamento de Matemática Aplicada, Universidad Politécnica de Madrid, C/ José Antonio Novais 10, \\ 28040 Madrid, Spain \\ 2 Departamento de Matemáticas - I.U.M.A., Universidad de Zaragoza, Campus Plaza San Francisco, \\ 50009 Zaragoza, Spain; ugarte@unizar.es \\ 3 Centro Universitario de la Defensa - I.U.M.A., Academia General Militar, Crta. de Huesca s/n, \\ 50090 Zaragoza, Spain; raquelvg@unizar.es \\ * Correspondence: adela.latorre@upm.es
}

Received: 26 August 2020; Accepted: 8 September 2020; Published: 11 September 2020

Abstract: We prove that for any $n \geq 4$, there are infinitely many real homotopy types of $2 n$-dimensional nilmanifolds admitting generalized complex structures of every type $k$, for $0 \leq k \leq n$.

Keywords: nilmanifold; nilpotent Lie algebra; complex structure; symplectic form; generalized complex structure; homotopy theory; minimal model

\section{Introduction}

Nilmanifolds constitute a well-known class of compact manifolds providing interesting explicit examples of geometric structures with special properties. A nilmanifold is a compact quotient $N=\Gamma \backslash G$ of a connected and simply connected nilpotent Lie group $G$ by a lattice $\Gamma$ of maximal rank in $G$. Hence, any left-invariant geometric structure on $G$ descends to $N$. We will refer to such structures as invariant. For instance, there are nilmanifolds admitting invariant complex structures, as the Iwasawa manifold, or invariant symplectic forms, as the Kodaira-Thurston manifold, with remarkable properties [1,2]. However, by [3], a nilmanifold cannot admit any Kähler metric (invariant or not), unless it is a torus. Since there are also nilmanifolds with no invariant complex structures or symplectic forms, it is an interesting problem to understand which nilmanifolds do admit such kinds of structures.

Symplectic and complex geometries constitute two special cases in the unified framework given by generalized complex geometry, introduced by Hitchin in [4] and further developed by Gualtieri [5]. In [6], Cavalcanti and Gualtieri study invariant generalized complex structures on nilmanifolds. Furthermore, Angella, Calamai and Kasuya show in [7] that nilmanifolds provide a nice class for investigating cohomological aspects of generalized complex structures.

In ([6], Theorem 3.1) the authors prove that any invariant generalized complex structure on a $2 n$-dimensional nilmanifold must be generalized Calabi-Yau, extending a result of Salamon [8] for invariant complex structures. This means that any generalized complex structure is given by a (left-invariant) trivialization $\rho$ of the canonical bundle, i.e.,

$$
\rho=e^{B+i \omega} \Omega,
$$

where $B, \omega$ are real invariant 2 -forms and $\Omega$ is a globally decomposable complex $k$-form, i.e., $\Omega=\theta^{1} \wedge \cdots \wedge \theta^{k}$, where each $\theta^{i}$ is an invariant 1 -form. Moreover, these data satisfy the non-degeneracy condition

$$
\omega^{n-k} \wedge \Omega \wedge \bar{\Omega} \neq 0,
$$


as well as the integrability condition

$$
d \rho=0
$$

The integer $0 \leq k \leq n$ is called the type of the generalized complex structure. Type $k=n$ corresponds to usual complex structures, whereas structures of type $k=0$ are symplectic. It is proved in [6] that every 6-dimensional nilmanifold admits a generalized complex structure of type $k$, for at least one $0 \leq k \leq 3$; however, it is shown that there are nilmanifolds in eight dimensions not admitting any invariant generalized complex structure.

Let $\mathfrak{g}$ be the nilpotent Lie algebra underlying the nilmanifold $N=\Gamma \backslash G$, and let $\left(\wedge \mathfrak{g}^{*}, d\right)$ be the Chevalley-Eilenberg complex seen as a commutative differential graded algebra (CDGA). Hasegawa proved in [3] that this CDGA provides not only the $\mathbb{R}$-minimal model of $N$ but also its $\mathbb{Q}$-minimal model. A result by Bazzoni and Muñoz asserts that, in six dimensions, there are infinitely many rational homotopy types of nilmanifolds, but only 34 different real homotopy types (see [9], Theorem 2). Hence, there only exits a finite number of real homotopy types of 6-dimensional nilmanifolds admitting any kind of geometric structure. The existence of infinitely many real homotopy types of 8-dimensional nilmanifolds with a complex structure (having special Hermitian metrics) is proved in [10]. However, these nilmanifolds do not admit any symplectic form. For this reason, we address the problem of finding a family of nilmanifolds with infinitely many real homotopy types that admit not only complex and symplectic structures, but also generalized complex structures of every possible type. In particular, we prove the following:

Theorem 1. There are infinitely many real homotopy types of 8-dimensional nilmanifolds admitting generalized complex structures of every type $k$, for $0 \leq k \leq 4$.

Although in dimension 6 there are nilmanifolds admitting generalized complex structures of every possible type, their real homotopy types are finite. As our result shows, this does no longer hold in higher dimensions.

This paper is structured as follows. In Section 2, we review some general results about minimal models and homotopy theory, and we define a family of nilmanifolds $N_{\alpha}$ in eight dimensions depending on a rational parameter $\alpha>0$. Section 3 is devoted to the construction of generalized complex structures on the nilmanifolds $N_{\alpha}$. More concretely, we prove the following:

Proposition 1. For each $\alpha \in \mathbb{Q}^{+}$, the 8-dimensional nilmanifold $N_{\alpha}$ has generalized complex structures of type $k$, for every $0 \leq k \leq 4$.

In Section 4, we study the real homotopy types of the nilmanifolds in the family $\left\{N_{\alpha}\right\}_{\alpha \in \mathbb{Q}^{+}}$. More precisely, the result below is attained:

Proposition 2. If $\alpha \neq \alpha^{\prime}$, then the nilmanifolds $N_{\alpha}$ and $N_{\alpha^{\prime}}$ have non-isomorphic $\mathbb{R}$-minimal models, so they have different real homotopy types.

Note that Theorem 1 is a direct consequence of Propositions 1 and 2 . Moreover, by taking products with even dimensional tori, the result holds in any dimension $2 n \geq 8$ and for every $0 \leq k \leq n$. Furthermore, our result in Theorem 1 can be extended to the complex homotopy setting (see Remark 2 for details). Since the nilmanifolds $N_{\alpha}$ cannot admit any Kähler metric [3], one has the following:

Corollary 1. Let $n \geq 4$. There are infinitely many complex homotopy types of $2 n$-dimensional compact non-Kähler manifolds admitting generalized complex structures of every type $k$, for $0 \leq k \leq n$. 


\section{The family of Nilmanifolds $N_{\alpha}$}

Let us start recalling some general results about homotopy theory and minimal models, with special attention to the class of nilmanifolds. In [11], Sullivan shows that it is possible to associate a minimal model to any nilpotent $C W$-complex $X$, i.e., a space $X$ whose fundamental group $\pi_{1}(X)$ is a nilpotent group that acts in a nilpotent way on the higher homotopy group $\pi_{k}(X)$ of $X$ for every $k>1$. Recall that a minimal model is a commutative differential graded algebra, CDGA for short, $\left(\wedge V_{X}, d\right)$ defined over the rational numbers $\mathbb{Q}$ and satisfying a certain minimality condition, that encodes the rational homotopy type of $X$ [12].

More generally, let $\mathbb{K}$ be the field $\mathbb{Q}$ or $\mathbb{R}$. A CDGA $(\wedge V, d)$ defined over $\mathbb{K}$ is said to be minimal if the following conditions hold:

(i) $\wedge V$ is the free commutative algebra generated by the graded vector space $V=\oplus V^{l}$;

(ii) there exists a basis $\left\{x_{j}\right\}_{j \in J}$, for some well-ordered index set $J$, such that $\operatorname{deg}\left(x_{k}\right) \leq \operatorname{deg}\left(x_{j}\right)$

for $k<j$, and each $d x_{j}$ is expressed in terms of the preceding $x_{k}(k<j)$.

A $\mathbb{K}$-minimal model of a differentiable manifold $M$ is a minimal CDGA $(\wedge V, d)$ over $\mathbb{K}$ together with a quasi-isomorphism $\phi$ from $(\Lambda V, d)$ to the $\mathbb{K}$-de Rham complex of $M$, i.e., a morphism $\phi$ inducing an isomorphism in cohomology. Here, the $\mathbb{K}$-de Rham complex of $M$ is the usual de Rham complex of differential forms $\left(\Omega^{*}(M), d\right)$ when $\mathbb{K}=\mathbb{R}$, whereas for $\mathbb{K}=\mathbb{Q}$ one considers $\mathbb{Q}$-polynomial forms instead. Notice that the $\mathbb{K}$-minimal model is unique up to isomorphism, since char $(\mathbb{K})=0$. By $[11,13]$, two nilpotent manifolds $M_{1}$ and $M_{2}$ have the same $\mathbb{K}$-homotopy type if and only if their $\mathbb{K}$-minimal models are isomorphic. It is clear that if $M_{1}$ and $M_{2}$ have different real homotopy types, then $M_{1}$ and $M_{2}$ also have different rational homotopy types.

Let $N$ be a nilmanifold, i.e., $N=\Gamma \backslash G$ is a compact quotient of a connected and simply connected nilpotent Lie group $G$ by a lattice $\Gamma$ of maximal rank. For any nilmanifold $N$, one has $\pi_{1}(N)=\Gamma$, which is nilpotent, and $\pi_{k}(N)=0$ for every $k \geq 2$. Therefore, nilmanifolds are nilpotent spaces.

Let $n$ be the dimension of the nilmanifold $N=\Gamma \backslash G$, and let $\mathfrak{g}$ be the Lie algebra of $G$. It is well known that the minimal model of $N$ is given by the Chevalley-Eilenberg complex $\left(\wedge \mathfrak{g}^{*}, d\right)$ of $\mathfrak{g}$. Recall that by [14], the existence of a lattice $\Gamma$ of maximal rank in $G$ is equivalent to the nilpotent Lie algebra $\mathfrak{g}$ being rational, i.e., there exists a basis $\left\{e^{1}, \ldots, e^{n}\right\}$ for the dual $\mathfrak{g}^{*}$ such that the structure constants are rational numbers. Thus, the rational and the nilpotency conditions of the Lie algebra $\mathfrak{g}$ allow to take a basis $\left\{e^{1}, \ldots, e^{n}\right\}$ for $\mathfrak{g}^{*}$ satisfying

$$
d e^{1}=d e^{2}=0, \quad d e^{j}=\sum_{i, k<j} a_{i k}^{j} e^{i} \wedge e^{k} \text { for } j=3, \ldots, n,
$$

with structure constants $a_{i k}^{j} \in \mathbb{Q}$.

Therefore, $\left(\wedge \mathfrak{g}^{*}, d\right)$ is a CDGA satisfying both conditions (i) and (ii) with ordered index set $J=\{1, \ldots, n\}$ and $V=V^{1}=\left\langle x_{1}, \ldots, x_{n}\right\rangle=\sum_{j=1}^{n} \mathbb{Q} x_{j}$, where $x_{j}=e^{j}$ for $1 \leq j \leq n$. That is to say, the CDGA $\left(\wedge \mathfrak{g}^{*}, d\right)$ over $\mathbb{Q}$ is minimal, and it is determined by

$$
\left(\bigwedge\left\langle x_{1}, \ldots, x_{n}\right\rangle, d\right)
$$

with $n$ generators $x_{1}, \ldots, x_{n}$ of degree 1 satisfying equations of the form (4). Notice that the CDGA $\left(\wedge \mathfrak{g}^{*}, d\right)$ over $\mathbb{R}$ is also minimal, since it is given by

$$
\left(\bigwedge\left\langle x_{1}, \ldots, x_{n}\right\rangle \otimes \mathbb{R}, d\right)
$$

There is a canonical morphism $\phi$ from the Chevalley-Eilenberg complex $\left(\bigwedge \mathfrak{g}^{*}, d\right)$ to the de Rham complex $\left(\Omega^{*}(\Gamma \backslash G), d\right)$ of the nilmanifold. Nomizu proves in [15] that $\phi$ induces an isomorphism in cohomology, so the $\mathbb{R}$-minimal model of the nilmanifold $N=\Gamma \backslash G$ is given by (6). Hasegawa observes 
in [3] that (5) is the $\mathbb{Q}$-minimal model of $N$ and that, conversely, given a $\mathbb{Q}$-minimal CDGA of the form (5), there exists a nilmanifold $N$ with (5) as its $\mathbb{Q}$-minimal model.

Deligne, Griffiths, Morgan and Sullivan prove in [13] that the $\mathbb{K}$-minimal model, $\mathbb{K}=\mathbb{Q}$ or $\mathbb{R}$, of a compact Kähler manifold is formal, i.e., it is quasi-isomorphic to its cohomology. Hasegawa shows in [3] that the minimal model (5) is formal if and only if all the structure constants $a_{i k}^{j}$ in (4) vanish, so a symplectic nilmanifold does not admit any Kähler metric unless it is a torus. (See for instance $[2,16]$ for more results on homotopy theory and applications to symplectic geometry.)

Bazzoni and Muñoz study in [9] the $\mathbb{K}$-homotopy types of nilmanifolds of low dimension. They prove that, up to dimension 5, the number of rational homotopy types of nilmanifolds is finite. However, in six dimensions the following result holds:

Theorem 2. ([9], Theorem 2) There are infinitely many rational homotopy types of 6-dimensional nilmanifolds, but there are only 34 real homotopy types of 6-dimensional nilmanifolds.

As a direct consequence, there is only a finite number of real homotopy types of 6-dimensional nilmanifolds admitting an extra geometric structure of any kind (in particular, generalized complex structures; see Section 3 for definition). We will prove that, in contrast to the 6-dimensional case, there are infinitely many real homotopy types of 8-dimensional nilmanifolds admitting generalized complex structures of every type $k$, for $0 \leq k \leq 4$.

To construct such nilmanifolds, let us take a positive rational number $\alpha$ and consider the connected, simply connected, nilpotent Lie group $G_{\alpha}$ corresponding to the nilpotent Lie algebra $\mathfrak{g}_{\alpha}$ defined by

$$
\left\{\begin{array}{l}
d e^{1}=d e^{2}=d e^{3}=d e^{4}=0, \\
d e^{5}=e^{12} \\
d e^{6}=e^{15}+(1-\alpha) e^{24} \\
d e^{7}=-(1+\alpha) e^{14}-e^{23}+(1+\alpha) e^{25}, \\
d e^{8}=e^{16}+e^{27}+e^{34}-2 e^{45},
\end{array}\right.
$$

where $e^{i j}=e^{i} \wedge e^{j}$, being $\left\{e^{i}\right\}_{i=1}^{8}$ a basis for $\mathfrak{g}_{\alpha}^{*}$, and $\alpha \in \mathbb{Q}^{+}$. It is clear from (7) that the Lie algebra $\mathfrak{g}_{\alpha}$ is rational, hence by the Mal'cev theorem [14], there exists a lattice $\Gamma_{\alpha}$ of maximal rank in $G_{\alpha}$. We denote by $N_{\alpha}=\Gamma_{\alpha} \backslash G_{\alpha}$ the corresponding compact quotient.

Therefore, we have defined a family of nilmanifolds $N_{\alpha}$ of dimension 8 depending on the rational parameter $\alpha \in \mathbb{Q}^{+}$. We will study the properties of $N_{\alpha}$, for $\alpha \in \mathbb{Q}^{+}$, in Sections 3 and 4 . Here, we simply provide their Betti numbers.

A direct calculation using Nomizu's theorem [15] allows to explicitly compute the de Rham cohomology groups of any nilmanifold $N_{\alpha}$. In particular, for degrees $1 \leq l \leq 3$, the $l$-th de Rham cohomology groups $H_{\mathrm{dR}}^{l}\left(N_{\alpha}\right)$ are

$$
\begin{aligned}
H_{\mathrm{dR}}^{1}\left(N_{\alpha}\right)=\langle & \left.\left\langle e^{1}\right],\left[e^{2}\right],\left[e^{3}\right],\left[e^{4}\right]\right\rangle, \\
H_{\mathrm{dR}}^{2}\left(N_{\alpha}\right)=\left\langle\left[e^{13}\right],\left[e^{14}\right],\left[e^{23}\right],\left[e^{24}\right],\left[e^{34}\right],\left[e^{16}-(1-\alpha) e^{45}\right],\right. & {\left.\left[e^{17}+(1+\alpha) e^{26}+e^{35}\right],\left[(1+\alpha) e^{18}-e^{37}-(1+\alpha)(3+\alpha) e^{46}+(1+\alpha) e^{57}\right]\right\rangle, } \\
H_{\mathrm{dR}}^{3}\left(N_{\alpha}\right)=\left\langle\left[e^{127}\right],\left[e^{146}\right],\left[e^{256}\right],\left[e^{248}+e^{456}\right],\left[e^{128}+2 e^{246}-e^{345}\right],\left[e^{136}+(1-\alpha) e^{345}\right],\right. & {\left[e^{137}+(1+\alpha) e^{236}\right],\left[e^{156}-(1-\alpha) e^{246}\right],\left[e^{138}+(3+\alpha) e^{346}-e^{357}\right], } \\
& {\left[e^{147}+(1+\alpha) e^{246}+e^{345}\right],\left[(1+\alpha) e^{148}-e^{347}-(1+\alpha) e^{457}\right], } \\
& {\left.\left[e^{238}+e^{356}+(3-\alpha)\left(e^{148}-e^{457}\right)\right]\right\rangle . }
\end{aligned}
$$


Let $b_{l}\left(N_{\alpha}\right)$ denote the $l$-th Betti number of $N_{\alpha}$. By duality we have:

$$
b_{0}\left(N_{\alpha}\right)=b_{8}\left(N_{\alpha}\right)=1, \quad b_{1}\left(N_{\alpha}\right)=b_{7}\left(N_{\alpha}\right)=4, \quad b_{2}\left(N_{\alpha}\right)=b_{6}\left(N_{\alpha}\right)=8, \quad b_{3}\left(N_{\alpha}\right)=b_{5}\left(N_{\alpha}\right)=12 .
$$

One can finally compute the Betti number $b_{4}\left(N_{\alpha}\right)$ taking into account that the Euler-Poincaré characteristic $\chi$ of a nilmanifold always vanishes, namely,

$$
0=\chi\left(N_{\alpha}\right)=\sum_{l=0}^{8}(-1)^{l} b_{l}\left(N_{\alpha}\right)=b_{4}\left(N_{\alpha}\right)+2\left(b_{0}\left(N_{\alpha}\right)-b_{1}\left(N_{\alpha}\right)+b_{2}\left(N_{\alpha}\right)-b_{3}\left(N_{\alpha}\right)\right),
$$

which implies $b_{4}\left(N_{\alpha}\right)=14$. In particular, we observe that the Betti numbers of the nilmanifolds $N_{\alpha}$ do not depend on $\alpha$.

\section{Generalized Complex Structures on the Nilmanifolds $N_{\alpha}$}

Generalized complex geometry, in the sense of Hitchin and Gualtieri [4,5], establishes a unitary framework for symplectic and complex geometries. Let $M$ be a compact differentiable manifold of dimension $2 n$. Denote by $T M$ the tangent bundle and by $T^{*} M$ the cotangent bundle, and consider the vector bundle $T M \oplus T^{*} M$ endowed with the natural symmetric pairing

$$
\langle X+\xi \mid Y+\eta\rangle=\frac{1}{2}(\xi(Y)+\eta(X)) .
$$

Recall that the Courant bracket on the space $\mathcal{C}^{\infty}\left(T M \oplus T^{*} M\right)$ is given by

$$
[X+\xi, Y+\eta]=[X, Y]+\mathcal{L}_{X} \eta-\mathcal{L}_{Y} \xi-\frac{1}{2} d\left(\iota_{X} \eta-\iota_{Y} \xi\right)
$$

where $\mathcal{L}$ and $\iota$ respectively denote the Lie derivative and the interior product. A generalized complex structure on $M$ is an endomorphism $\mathcal{J} \in \operatorname{End}\left(T M \oplus T^{*} M\right)$ satisfying $\mathcal{J}^{2}=-1$ whose $i$-eigenbundle $L \subset\left(T M \oplus T^{*} M\right) \otimes \mathbb{C}$ is involutive with respect to the Courant bracket.

There is an action of $T M \oplus T^{*} M$ on $\wedge^{\bullet} T^{*} M$ given by

$$
(X+\xi) \cdot \rho=\iota_{X} \rho+\xi \wedge \rho .
$$

Now, for a generalized complex structure $\mathcal{J}$ with $i$-eigenbundle $L$, one can define the canonical line bundle $K \subset \wedge^{\bullet} T^{*} M \otimes \mathbb{C}$ as

$$
L=\operatorname{Ann}(K)=\left\{u \in\left(T M \oplus T^{*} M\right) \otimes \mathbb{C} \mid u \cdot K=0\right\} .
$$

Any $\rho \in K$ is a non-degenerate pure form, i.e., it can be written as

$$
\rho=e^{B+i \omega} \Omega
$$

where $B, \omega$ are real 2-forms and $\Omega=\theta^{1} \wedge \cdots \wedge \theta^{k}$ is a complex decomposable $k$-form, such that

$$
\omega^{n-k} \wedge \Omega \wedge \bar{\Omega} \neq 0
$$

The number $k$ is called the type of the generalized complex structure. Moreover, any $\phi \in \mathcal{C}^{\infty}(K)$ is integrable, i.e., there exists $X+\xi \in \mathcal{C}^{\infty}\left(T M \oplus T^{*} M\right)$ satisfying

$$
d \phi=(X+\xi) \cdot \phi
$$


Notice that the converse also holds: if $K \subset \wedge^{\bullet} T^{*} M \otimes \mathbb{C}$ is a line bundle such that any $\rho \in K$ is a non-degenerate pure form and any $\phi \in \mathcal{C}^{\infty}(K)$ is integrable, then we have a generalized complex structure whose $i$-eigenbundle is $L=\operatorname{Ann}(K)$.

In the case that $K$ is a trivial bundle admitting a nowhere vanishing closed section, the generalized complex structure is called generalized Calabi-Yau.

Recall that if $J$ is a complex structure on $M$ then

$$
\mathcal{J}_{J}=\left(\begin{array}{cc}
-J & 0 \\
0 & J^{*}
\end{array}\right)
$$

is a generalized complex structure of type $n$, and if $\omega$ is a symplectic form on $M$ then

$$
\mathcal{J}_{\omega}=\left(\begin{array}{cc}
0 & -\omega^{-1} \\
\omega & 0
\end{array}\right)
$$

is a generalized complex structure of type 0 . Near a regular point (i.e., a point where the type is locally constant), a generalized complex structure is equivalent to a product of a complex and a symplectic structure ([5], Theorem 3.6).

In the case of a nilmanifold N, Cavalcanti and Gualtieri proved in ([6], Theorem 3.1) that any invariant generalized complex structure on $N$ must be generalized Calabi-Yau. Hence, it is given by a (left-invariant) trivialization $\rho$ of the canonical bundle of the form (1) satisfying the non-degeneracy condition (2) and the integrability condition (3).

Let us now prove Proposition 1 , that is, each nilmanifold $N_{\alpha}$ has generalized complex structures of every type $k$, for $0 \leq k \leq 4$. These structures will be explicitly described in terms of the global basis of invariant 1-forms $\left\{e^{i}\right\}_{i=1}^{8}$ on $N_{\alpha}$ given in (7). We begin providing a structure of type 4 .

- Generalized complex structure of type 4 (complex structure). We define the following complex 1-forms:

$$
\begin{aligned}
\theta^{1} & =\frac{1}{2}\left(\frac{1}{\sqrt{3+\alpha}} e^{1}-e^{2}\right)+\frac{i}{2}\left(\frac{1}{\sqrt{3+\alpha}} e^{1}+e^{2}\right) \\
\theta^{2} & =-\alpha e^{4}-\frac{i}{\sqrt{3+\alpha}}\left(\frac{1}{2} e^{3}+e^{5}\right) \\
\theta^{3} & =\frac{\alpha}{(1+\alpha)^{2}}\left(e^{6}-\frac{1}{\sqrt{3+\alpha}} e^{7}\right)+\frac{i \alpha}{(1+\alpha)^{2}}\left(e^{6}+\frac{1}{\sqrt{3+\alpha}} e^{7}\right) \\
\theta^{4} & =\frac{1}{\sqrt{3+\alpha}}\left(e^{5}+\frac{1-\alpha}{2(1+\alpha)^{2}} e^{3}\right)-i \alpha\left(e^{4}+\frac{2}{(1+\alpha)^{2} \sqrt{3+\alpha}} e^{8}\right) .
\end{aligned}
$$

From Equation (7), we get

$$
\left\{\begin{array}{l}
d \theta^{1}=0 \\
d \theta^{2}=\theta^{1} \wedge \overline{\theta^{1}} \\
d \theta^{3}=\theta^{1} \wedge \theta^{4}+\theta^{1} \wedge \overline{\theta^{4}}+\frac{2 \alpha}{(1+\alpha)^{2}} \theta^{2} \wedge \overline{\theta^{1}}+\frac{2 i}{(1+\alpha)^{2}} \theta^{1} \wedge \overline{\theta^{2}} \\
d \theta^{4}=i \theta^{1} \wedge \overline{\theta^{1}}-\frac{2}{(1+\alpha)^{2}} \theta^{2} \wedge \overline{\theta^{2}}-i \theta^{1} \wedge \overline{\theta^{3}}+i \theta^{3} \wedge \overline{\theta^{1}} .
\end{array}\right.
$$

Declaring the forms $\theta^{i}$ to be of bidegree $(1,0)$, we obtain an almost complex structure $J$ on the nilmanifold $N_{\alpha}$ for every $\alpha \in \mathbb{Q}^{+}$. It follows from (9) that $d \theta^{i}$ has no $(0,2)$ component, so $J$ is integrable. Hence, $\rho=\Omega=\theta^{1} \wedge \theta^{2} \wedge \theta^{3} \wedge \theta^{4}$ is a generalized complex structure of type 4 . 
Remark 1. The complex nilmanifold $\left(N_{\alpha}, J\right)$ has a holomorphic Poisson structure given by the holomorphic bivector $\beta=X_{3} \wedge X_{4}$ of rank two, where $\left\{X_{i}\right\}$ is the dual basis of $\left\{\theta^{i}\right\}$ (see [6], (Theorem 5.1) for the existence of such a bivector on nilmanifolds). It is worth observing that $\left(N_{\alpha}, J\right)$ does not admit any (invariant or not) holomorphic symplectic structure: since the center of the Lie algebra $\mathfrak{g}_{\alpha}$ has dimension 1 , the complex structure defined by (8) is strongly non-nilpotent (see [17] for properties on this kind of complex structure); by [18], a strongly non-nilpotent complex structure on an 8-dimensional nilmanifold cannot support any holomorphic symplectic form.

- Generalized complex structure of type 3. Let us consider $\rho=e^{B+i \omega} \Omega$, with $B=0, \omega=i \theta^{4} \wedge \overline{\theta^{4}}$ and $\Omega=\theta^{1} \wedge \theta^{2} \wedge \theta^{3}$, where $\theta^{1}, \theta^{2}, \theta^{3}$ and $\theta^{4}$ are the complex 1 -forms given in (8). It is clear that

$$
\omega \wedge \Omega \wedge \bar{\Omega}=-i \theta^{1} \wedge \theta^{2} \wedge \theta^{3} \wedge \theta^{4} \wedge \overline{\theta^{1}} \wedge \overline{\theta^{2}} \wedge \overline{\theta^{3}} \wedge \overline{\theta^{4}} \neq 0,
$$

so the non-degeneracy condition (2) is satisfied. A direct calculation using (9) shows

$$
d\left(\theta^{1} \wedge \theta^{2} \wedge \theta^{3}\right)=0
$$

and

$$
d \omega \wedge \theta^{1} \wedge \theta^{2} \wedge \theta^{3}=i d \theta^{4} \wedge \overline{\theta^{4}} \wedge \theta^{1} \wedge \theta^{2} \wedge \theta^{3}-i \theta^{4} \wedge d \overline{\theta^{4}} \wedge \theta^{1} \wedge \theta^{2} \wedge \theta^{3}=0,
$$

which implies that $d \rho=0$, i.e., the integrability condition (3) holds.

Therefore, the nilmanifolds $N_{\alpha}$ have generalized complex structures of type 3 .

- Generalized complex structure of type 2. Recall that the action of a bivector $\beta$ is given by $\rho \mapsto e^{\iota} \beta$. If $J$ is a complex structure and $\beta$ is a holomorphic Poisson structure of rank $l$, then one can deform $J$ into a generalized complex structure of type $n-l$ (see [5]). In ([6], Theorem 5.1) it is proved that every invariant complex structure on a $2 n$-dimensional nilmanifold can be deformed, via such a $\beta$-field with $l=2$, to get an invariant generalized complex structure of type $n-2$. Therefore, our nilmanifolds have a generalized complex structure of type 2 .

More concretely, in view of Remark 1 , from the generalized complex structure of type 4 defined by $\rho=\theta^{1} \wedge \theta^{2} \wedge \theta^{3} \wedge \theta^{4}$ above, we get that

$$
\widetilde{\rho}=e^{\theta^{3} \wedge \theta^{4}} \Omega,
$$

with $\Omega=\theta^{1} \wedge \theta^{2}$, is a generalized complex structure of type 2. Indeed, $B=\frac{1}{2}\left(\theta^{3} \wedge \theta^{4}+\overline{\theta^{3}} \wedge \overline{\theta^{4}}\right)$ and $\omega=-\frac{i}{2}\left(\theta^{3} \wedge \theta^{4}-\overline{\theta^{3}} \wedge \overline{\theta^{4}}\right)$. Thus,

$$
\omega^{2} \wedge \Omega \wedge \bar{\Omega}=\frac{1}{2} \theta^{3} \wedge \theta^{4} \wedge \overline{\theta^{3}} \wedge \overline{\theta^{4}} \wedge \theta^{1} \wedge \theta^{2} \wedge \overline{\theta^{1}} \wedge \overline{\theta^{2}} \neq 0,
$$

and $d\left(\theta^{1} \wedge \theta^{2}\right)=0$ and $d(B+i \omega) \wedge \theta^{1} \wedge \theta^{2}=d\left(\theta^{3} \wedge \theta^{4}\right) \wedge \theta^{1} \wedge \theta^{2}=d\left(\theta^{1} \wedge \theta^{2} \wedge \theta^{3} \wedge \theta^{4}\right)=0$ by the Equation (9).

For the definition of generalized complex structures of type 1 and type 0 we will deal with the space $Z^{2}\left(N_{\alpha}\right)$ of invariant closed 2-forms on the nilmanifold $N_{\alpha}$. The following lemma is straightforward:

Lemma 1. Every invariant closed 2 -form $\omega$ on the nilmanifold $N_{\alpha}$ is given by

$$
\begin{aligned}
\omega= & x_{12} e^{12}+x_{13} e^{13}+x_{14} e^{14}+x_{15} e^{15}+x_{16} e^{16}+x_{17} e^{17}-(1+\alpha) x_{37} e^{18} \\
& +x_{23} e^{23}+x_{24} e^{24}+x_{25} e^{25}+(1+\alpha) x_{17} e^{26}+x_{27} e^{27}+x_{34} e^{34}+x_{17} e^{35}+x_{37} e^{37} \\
& -\left((1-\alpha) x_{16}+(1+\alpha) x_{27}\right) e^{45}+(1+\alpha)(3+\alpha) x_{37} e^{46}-(1+\alpha) x_{37} e^{57},
\end{aligned}
$$


where $x_{12}, \ldots, x_{37} \in \mathbb{R}$. Hence, the space $Z^{2}\left(N_{\alpha}\right)$ has dimension 12 .

- Generalized complex structure of type 1 . We must find $\rho=e^{B+i \omega} \Omega$, with $B, \omega$ real invariant 2-forms and $\Omega$ a complex 1-form, satisfying

$$
\omega^{3} \wedge \Omega \wedge \bar{\Omega} \neq 0, \quad d \Omega=0, \quad(d B+i d \omega) \wedge \Omega=0
$$

Since $\Omega$ is a complex 1 -form, it can be written as $\Omega=\sum_{j=1}^{4} z_{j} e^{j}$, for some complex coefficients $z_{1}, \ldots, z_{4} \in \mathbb{C}$. Let us choose $\Omega=e^{3}+i e^{4}$, which satisfies $d \Omega=0$ according to the structure Equation (7).

We consider $B=0$ and let $\omega$ be any 2 -form given in (10) with coefficients $x_{17}, x_{37} \neq 0$. A direct calculation shows that

$$
\omega^{3} \wedge \Omega \wedge \bar{\Omega}=12 i(1+\alpha)^{3} x_{17} x_{37}^{2} e^{12345678} \neq 0
$$

Since both $B$ and $\omega$ are closed, the condition $(d B+i d \omega) \wedge \Omega=0$ is trivially satisfied, and $\rho=e^{i \omega} \Omega$ defines a generalized complex structure of type 1 on the nilmanifold $N_{\alpha}$.

- Generalized complex structure of type 0 (symplectic structure). The form $\omega$ in Lemma 1 determined by (10) satisfies

$$
\begin{aligned}
\omega^{4}=-24(1+\alpha)^{2} x_{37}^{2}( & \left((1-\alpha) x_{16}-2 x_{27}+(1+\alpha) x_{34}\right) x_{17} \\
& \left.+\left((1+\alpha)(3+\alpha) x_{23}+(3+\alpha) x_{25}\right) x_{37}\right) e^{12345678} .
\end{aligned}
$$

It suffices to choose, for instance, $x_{17}=x_{23}=0$ and $x_{25} x_{37} \neq 0$ to get a symplectic form $\omega$ on $N_{\alpha}$. This concludes the proof of Proposition 1.

\section{The Nilmanifolds $N_{\alpha}$ and Their Minimal Model}

The goal of this section is to prove Proposition 2, i.e., the nilmanifolds $N_{\alpha}$ and $N_{\alpha^{\prime}}$ have non-isomorphic $\mathbb{R}$-minimal models for $\alpha \neq \alpha^{\prime}$.

As we recalled in Section 2, the $\mathbb{R}$-minimal model of the nilmanifold $N_{\alpha}$ is given by the Chevalley-Eilenberg complex $\left(\wedge \mathfrak{g}_{\alpha}^{*}, d\right)$ of its underlying Lie algebra $\mathfrak{g}_{\alpha}$. Consequently, to prove Proposition 2, it suffices to show that the real Lie algebras $\mathfrak{g}_{\alpha}, \alpha \in \mathbb{Q}^{+}$, define a family of pairwise non-isomorphic nilpotent Lie algebras. Indeed, we will prove the following:

Proposition 3. If the nilpotent Lie algebras $\mathfrak{g}_{\alpha}$ and $\mathfrak{g}_{\alpha^{\prime}}$ are isomorphic, then $\alpha=\alpha^{\prime}$.

Remember that in eight dimensions, no classification of nilpotent Lie algebras is available. Indeed, nilpotent Lie algebras are classified only up to real dimension 7. More concretely, Gong classified in [19] the 7-dimensional nilpotent Lie algebras in 140 algebras together with 9 one-parameter families. One can check that our family $\left\{\mathfrak{g}_{\alpha}\right\}_{\alpha \in \mathbb{Q}^{+}}$is not an extension of any of those 9 families, i.e., the quotient of $\mathfrak{g}_{\alpha}$ by its center (which has dimension 1) does not belong to any of the 9 one-parameter families of Gong. Furthermore, the usual invariants for nilpotent Lie algebras are the same for all the algebras in the family $\left\{\mathfrak{g}_{\alpha}\right\}_{\alpha \in \mathbb{Q}^{+}}$. For instance, the dimensions of the terms in the ascending central series are $(1,3,6,8)$, whereas those of the descending central series are $(4,3,1,0)$ (see Lemma 3 for further details). Moreover, the dimensions of the Lie algebra cohomology groups $H^{k}\left(\mathfrak{g}_{\alpha}\right)$ coincide for every $\alpha \in \mathbb{Q}^{+}$, as shown at the end of Section 2. For this reason, we will directly analyze the existence of an isomorphism between any two of the nilpotent Lie algebras in our family $\left\{\mathfrak{g}_{\alpha}\right\}_{\alpha \in \mathbb{Q}^{+}}$.

The following technical lemma will be useful for our purpose. 
Lemma 2. Let $f: \mathfrak{g} \longrightarrow \mathfrak{g}^{\prime}$ be an isomorphism of the Lie algebras $\mathfrak{g}$ and $\mathfrak{g}^{\prime}$. Consider an ideal $\{0\} \neq \mathfrak{a} \subset \mathfrak{g}$, and let $\mathfrak{a}^{\prime}=f(\mathfrak{a}) \subset \mathfrak{g}^{\prime}$ be the corresponding ideal in $\mathfrak{g}^{\prime}$. Let $\left\{e_{r+1}, \ldots, e_{m}\right\}$, resp. $\left\{e_{r+1}^{\prime}, \ldots, e_{m}^{\prime}\right\}$, be a basis of $\mathfrak{a}$, resp. $\mathfrak{a}^{\prime}$, and complete it up to a basis $\left\{e_{1}, \ldots, e_{r}, e_{r+1}, \ldots, e_{m}\right\}$ of $\mathfrak{g}$, resp. $\left\{e_{1}^{\prime}, \ldots, e_{r}^{\prime}, e_{r+1}^{\prime}, \ldots, e_{m}^{\prime}\right\}$ of $\mathfrak{g}^{\prime}$. Denote the dual bases of $\mathfrak{g}^{*}$ and $\mathfrak{g}^{*}$ respectively by $\left\{e^{i}\right\}_{i=1}^{m}$ and $\left\{e^{\prime i}\right\}_{i=1}^{m}$. Then, the dual map $f^{*}: \mathfrak{g}^{*} \longrightarrow \mathfrak{g}^{*}$ satisfies

$$
f^{*}\left(e^{\prime i}\right) \wedge e^{1} \wedge \ldots \wedge e^{r}=0, \quad \text { for all } i=1, \ldots, r
$$

Proof. Let $\pi: \mathfrak{g} \longrightarrow \mathfrak{g} / \mathfrak{a}$ and $\pi^{\prime}: \mathfrak{g}^{\prime} \longrightarrow \mathfrak{g}^{\prime} / \mathfrak{a}^{\prime}$ be the natural projections, and $\tilde{f}: \mathfrak{g} / \mathfrak{a} \longrightarrow \mathfrak{g}^{\prime} / \mathfrak{a}^{\prime}$ the Lie algebra isomorphism induced by $f$ on the quotients. Taking the corresponding dual maps, we have the following commutative diagrams:
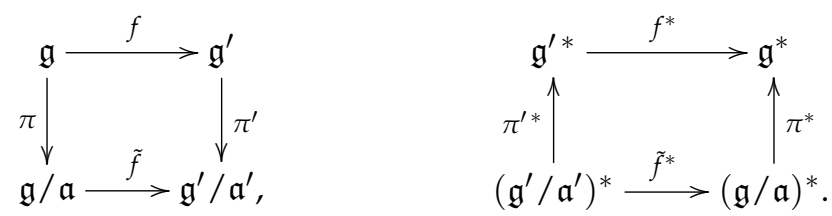

Taking the basis $\left\{e_{1}, \ldots, e_{r}, e_{r+1}, \ldots, e_{m}\right\}$ of $\mathfrak{g}$, we have that $\left\{\tilde{e}_{1}, \ldots, \tilde{e}_{r}\right\}$ is a basis of $\mathfrak{g} / \mathfrak{a}$. Let $\left\{\tilde{e}^{1}, \ldots, \tilde{e}^{r}\right\}$ be its dual basis for $(\mathfrak{g} / \mathfrak{a})^{*}$. Using a similar procedure, we find a basis $\left\{\tilde{e}^{\prime 1}, \ldots, \tilde{e}^{\prime r}\right\}$ for $\left(\mathfrak{g}^{\prime} / \mathfrak{a}^{\prime}\right)^{*}$. Since the maps $\pi^{*}$ and $\pi^{\prime *}$ are injective, and the diagram is commutative, we get

$$
f^{*}\left(e^{i}\right)=f^{*}\left(\pi^{*}\left(\tilde{e}^{\prime i}\right)\right)=\pi^{*}\left(\tilde{f}^{*}\left(\tilde{e}^{i}\right)\right) \in\left\langle e^{1}, \ldots, e^{r}\right\rangle,
$$

for any $1 \leq i \leq r$.

Applying the previous result to our particular case, we get:

Lemma 3. Consider $\mathfrak{g}_{\alpha}$ and $\mathfrak{g}_{\alpha^{\prime}}$ for $\alpha, \alpha^{\prime} \in \mathbb{Q}^{+}$. If $f: \mathfrak{g}_{\alpha} \longrightarrow \mathfrak{g}_{\alpha^{\prime}}$ is an isomorphism of Lie algebras, then in terms of their respective bases $\left\{e^{i}\right\}_{i=1}^{8}$ and $\left\{e^{\prime i}\right\}_{i=1}^{8}$ given in (7), the dual map $f^{*}: \mathfrak{g}_{\alpha^{\prime}}^{*} \longrightarrow \mathfrak{g}_{\alpha}^{*}$ satisfies

$$
\begin{array}{ll}
f^{*}\left(e^{\prime i}\right) \wedge e^{12}=0, \text { for } i=1,2, & f^{*}\left(e^{\prime 5}\right) \wedge e^{12345}=0, \\
f^{*}\left(e^{\prime i}\right) \wedge e^{1234}=0, \text { for } i=3,4, & f^{*}\left(e^{\prime i}\right) \wedge e^{1234567}=0, \text { for } i=6,7 .
\end{array}
$$

Proof. Recall that the ascending central series of a Lie algebra $\mathfrak{g}$ is defined by $\left\{\mathfrak{g}_{k}\right\}_{k}$, where $\mathfrak{g}_{0}=\{0\}$ and

$$
\mathfrak{g}_{k}=\left\{X \in \mathfrak{g} \mid[X, \mathfrak{g}] \subseteq \mathfrak{g}_{k-1}\right\}, \text { for } k \geq 1 .
$$

Observe that $\mathfrak{g}_{1}=Z(\mathfrak{g})$ is the center of $\mathfrak{g}$.

Let $\left\{e_{i}\right\}_{i=1}^{8}$ and $\left\{e_{i}^{\prime}\right\}_{i=1}^{8}$ be the bases for $\mathfrak{g}_{\alpha}$ and $\mathfrak{g}_{\alpha^{\prime}}$ dual to $\left\{e^{i}\right\}_{i=1}^{8}$ and $\left\{e^{\prime i}\right\}_{i=1}^{8}$, respectively. In terms of these bases, the ascending central series of $\mathfrak{g}_{\alpha}$ and $\mathfrak{g}_{\alpha^{\prime}}$ are

$$
\left(\mathfrak{g}_{\alpha}\right)_{1}=\left\langle e_{8}\right\rangle \subset\left(\mathfrak{g}_{\alpha}\right)_{2}=\left\langle e_{6}, e_{7}, e_{8}\right\rangle \subset\left(\mathfrak{g}_{\alpha}\right)_{3}=\left\langle e_{3}, e_{4}, e_{5}, e_{6}, e_{7}, e_{8}\right\rangle,
$$

and

$$
\left(\mathfrak{g}_{\alpha^{\prime}}\right)_{1}=\left\langle e_{8}^{\prime}\right\rangle \subset\left(\mathfrak{g}_{\alpha^{\prime}}\right)_{2}=\left\langle e_{6}^{\prime}, e_{7}^{\prime}, e_{8}^{\prime}\right\rangle \subset\left(\mathfrak{g}_{\alpha^{\prime}}\right)_{3}=\left\langle e_{3}^{\prime}, e_{4}^{\prime}, e_{5}^{\prime}, e_{6}^{\prime}, e_{7}^{\prime}, e_{8}^{\prime}\right\rangle
$$

Since $f\left(\left(\mathfrak{g}_{\alpha}\right)_{k}\right)=\left(\mathfrak{g}_{\alpha^{\prime}}\right)_{k}$ for any Lie algebra isomorphism $f: \mathfrak{g}_{\alpha} \longrightarrow \mathfrak{g}_{\alpha^{\prime}}$, applying Lemma 2 to the ideals $\mathfrak{a}=\left(\mathfrak{g}_{\alpha}\right)_{k}$ for $k=1,2,3$ one gets (12) for $i=1,2,5,6$ and 7 .

Moreover, the derived algebras of $\mathfrak{g}_{\alpha}$ and $\mathfrak{g}_{\alpha^{\prime}}$ are, respectively,

$$
\left[\mathfrak{g}_{\alpha}, \mathfrak{g}_{\alpha}\right]=\left\langle e_{5}, e_{6}, e_{7}, e_{8}\right\rangle, \quad\left[\mathfrak{g}_{\alpha^{\prime}}, \mathfrak{g}_{\alpha^{\prime}}\right]=\left\langle e_{5}^{\prime}, e_{6}^{\prime}, e_{7}^{\prime}, e_{8}^{\prime}\right\rangle
$$

Using again Lemma 2 with $\mathfrak{a}=\left[\mathfrak{g}_{\alpha}, \mathfrak{g}_{\alpha}\right]$, we obtain (12) for $i=3,4$. 
We are now in the conditions to prove Proposition 3.

Proof of Proposition 3. Given any homomorphism of Lie algebras $f: \mathfrak{g}_{\alpha} \longrightarrow \mathfrak{g}_{\alpha^{\prime}}$, its dual map $f^{*}: \mathfrak{g}_{\alpha^{\prime}}^{*} \longrightarrow \mathfrak{g}_{\alpha}^{*}$ naturally extends to a map $F: \Lambda^{*} \mathfrak{g}_{\alpha^{\prime}}^{*} \longrightarrow \bigwedge^{*} \mathfrak{g}_{\alpha}^{*}$ that commutes with the differentials, i.e., $F \circ d=d \circ F$. Hence, in terms of the bases $\left\{e^{i}\right\}_{i=1}^{8}$ for $\mathfrak{g}_{\alpha}^{*}$ and $\left\{e^{\prime i}\right\}_{i=1}^{8}$ for $\mathfrak{g}_{\alpha^{\prime}}^{*}$ satisfying the Equations (7) with respective parameters $\alpha$ and $\alpha^{\prime}$, any Lie algebra isomorphism is defined by

$$
F\left(e^{i}\right)=\sum_{j=1}^{8} \lambda_{j}^{i} e^{j}, \quad i=1, \ldots, 8,
$$

satisfying conditions

$$
d\left(F\left(e^{i}\right)\right)-F\left(d e^{i}\right)=0, \text { for each } 1 \leq i \leq 8,
$$

where the matrix $\Lambda=\left(\lambda_{j}^{i}\right)_{i, j=1, \ldots, 8}$ belongs to $\mathrm{GL}(8, \mathbb{R})$.

We first note that the preceding lemma allows us to simplify the $8 \times 8$ matrix $\Lambda$. In fact, from Lemma 3 one has that $\lambda_{j}^{i}=0$ for $1 \leq i \leq 2$ and $3 \leq j \leq 8$, for $3 \leq i \leq 5$ and $6 \leq j \leq 8$, and also $\lambda_{5}^{3}=\lambda_{5}^{4}=\lambda_{8}^{6}=\lambda_{8}^{7}=0$. Since $\Lambda$ belongs to $\mathrm{GL}(8, \mathbb{R})$, the previous conditions imply that $\lambda_{5}^{5} \neq 0$ and $\lambda_{8}^{8} \neq 0$.

Note also that (14) is trivially fulfilled for $1 \leq i \leq 4$. Hence, it suffices to focus on $5 \leq i \leq 8$. We will denote by $\left[d\left(F\left(e^{i}\right)\right)-F\left(d e^{\prime i}\right)\right]_{j r}$ the coefficient for $e^{j r}$ in the expression of the 2-form $d\left(F\left(e^{\prime i}\right)\right)-$ $F\left(d e^{\prime i}\right)$.

By a direct calculation we have

$$
0=\left[d\left(F\left(e^{\prime 8}\right)\right)-F\left(d e^{\prime 8}\right)\right]_{35}=2 \lambda_{3}^{4} \lambda_{5}^{5}
$$

Since $\lambda_{5}^{5} \neq 0$, we conclude that $\lambda_{3}^{4}=0$. Now observe that the following expressions must annihilate:

$$
\begin{aligned}
& {\left[d\left(F\left(e^{\prime 6}\right)\right)-F\left(d e^{\prime 6}\right)\right]_{23}=-\left(\lambda_{2}^{1} \lambda_{3}^{5}+\lambda_{7}^{6}\right)} \\
& {\left[d\left(F\left(e^{\prime 7}\right)\right)-F\left(d e^{\prime 7}\right)\right]_{23}=\lambda_{2}^{2}\left(\lambda_{3}^{3}-\left(1+\alpha^{\prime}\right) \lambda_{3}^{5}\right)-\lambda_{7}^{7}} \\
& {\left[d\left(F\left(e^{\prime 6}\right)\right)-F\left(d e^{\prime 6}\right)\right]_{25}=-\lambda_{2}^{1} \lambda_{5}^{5}+(1+\alpha) \lambda_{7}^{6}} \\
& {\left[d\left(F\left(e^{\prime 7}\right)\right)-F\left(d e^{\prime 7}\right)\right]_{25}=-\left(1+\alpha^{\prime}\right) \lambda_{2}^{2} \lambda_{5}^{5}+(1+\alpha) \lambda_{7}^{7} .}
\end{aligned}
$$

Solving $\lambda_{7}^{6}$ and $\lambda_{7}^{7}$ from the first two equations and replacing their values in the last ones, we get:

$$
\lambda_{2}^{1}\left(\lambda_{5}^{5}+(1+\alpha) \lambda_{3}^{5}\right)=0, \quad \lambda_{2}^{2}\left(\lambda_{3}^{3}-\left(1+\alpha^{\prime}\right) \lambda_{3}^{5}-\frac{1+\alpha^{\prime}}{1+\alpha} \lambda_{5}^{5}\right)=0 .
$$

Moreover, the following terms must vanish:

$$
\left[d\left(F\left(e^{\prime 8}\right)\right)-F\left(d e^{\prime 8}\right)\right]_{34}=-\lambda_{4}^{4}\left(\lambda_{3}^{3}+2 \lambda_{3}^{5}\right)+\lambda_{8}^{8}, \quad\left[d\left(F\left(e^{\prime 8}\right)\right)-F\left(d e^{\prime 8}\right)\right]_{45}=2\left(\lambda_{4}^{4} \lambda_{5}^{5}-\lambda_{8}^{8}\right) .
$$

From the second one, we have $\lambda_{8}^{8}=\lambda_{4}^{4} \lambda_{5}^{5}$. Since $\lambda_{8}^{8} \neq 0$, in particular also $\lambda_{4}^{4} \neq 0$. Using the first expression above, we can then solve

$$
\lambda_{3}^{3}=\lambda_{5}^{5}-2 \lambda_{3}^{5}
$$

In addition, observe that $\left[d\left(F\left(e^{\prime 5}\right)\right)-F\left(d e^{5}\right)\right]_{12}=\left[d\left(F\left(e^{\prime 7}\right)\right)-F\left(d e^{7}\right)\right]_{13}=0$ leads to

$$
\lambda_{5}^{5}=\lambda_{1}^{1} \lambda_{2}^{2}-\lambda_{2}^{1} \lambda_{1}^{2}, \quad \lambda_{1}^{2}\left(\lambda_{3}^{3}-\left(1+\alpha^{\prime}\right) \lambda_{3}^{5}\right)=0 .
$$

We now check that the vanishing of the coefficient $\lambda_{1}^{1}$ leads to a contradiction. Indeed, in such a case, the first expression in (17) becomes $\lambda_{5}^{5}=-\lambda_{2}^{1} \lambda_{1}^{2} \neq 0$, and from (15) we then have $\lambda_{5}^{5}=-(1+\alpha)$ $\lambda_{3}^{5}$, which plugged into (16) gives $\lambda_{3}^{3}=-(3+\alpha) \lambda_{3}^{5}$. Replacing this value in the second equation of (17), the condition $\lambda_{3}^{5}\left(\alpha+\alpha^{\prime}+4\right)=0$ arises. Since $\alpha$ and $\alpha^{\prime}$ are greater than zero, we are forced to consider 
$\lambda_{3}^{5}=0$. However, this leads to $\lambda_{5}^{5}=0$, which is a contradiction. Hence, we necessarily have that $\lambda_{1}^{1}$ is nonzero.

Since $\lambda_{1}^{1} \neq 0$, the condition

$$
0=\left[d\left(F\left(e^{\prime 6}\right)\right)-F\left(d e^{6}\right)\right]_{13}=-\lambda_{1}^{1} \lambda_{3}^{5}
$$

implies $\lambda_{3}^{5}=0$. Replacing this value in (15), (16), and (17), we obtain:

$$
\lambda_{2}^{1} \lambda_{5}^{5}=0, \quad \lambda_{1}^{2} \lambda_{5}^{5}=0, \quad \lambda_{2}^{2} \lambda_{5}^{5}\left(1-\frac{1+\alpha^{\prime}}{1+\alpha}\right)=0, \quad \lambda_{3}^{3}=\lambda_{5}^{5}=\lambda_{1}^{1} \lambda_{2}^{2}-\lambda_{2}^{1} \lambda_{1}^{2} .
$$

As $\lambda_{5}^{5} \neq 0$, one immediately has $\lambda_{1}^{2}=\lambda_{2}^{1}=0$ and $\lambda_{5}^{5}=\lambda_{1}^{1} \lambda_{2}^{2}$. Consequently $\lambda_{2}^{2} \neq 0$, which allows us to conclude $1+\alpha=1+\alpha^{\prime}$, and thus $\alpha=\alpha^{\prime}$. This completes the proof of the proposition.

Remark 2. In addition to the notions of rational and real homotopy types, there is also the notion of complex homotopy type [13]. Two manifolds $X$ and $Y$ have the same $\mathbb{C}$-homotopy type if and only if their $\mathbb{C}$-minimal models $\left(\wedge V_{X} \otimes_{\mathbb{Q}} \mathbb{C}, d\right)$ and $\left(\wedge V_{Y} \otimes_{\mathbb{Q}} \mathbb{C}, d\right)$ are isomorphic. Here, $\left(\wedge V_{X}, d\right)$ and $\left(\wedge V_{Y}, d\right)$ are the rational minimal models of $X$ and $Y$, respectively. Recall that when the field $\mathbb{K}$ has $\operatorname{char}(\mathbb{K})=0$, the $\mathbb{K}$-minimal model is unique up to isomorphism. Clearly, if $X$ and $Y$ have different complex homotopy types, then $X$ and $Y$ have different real (hence, also rational) homotopy types.

For nilmanifolds, it is proved in ([9], Theorem 2) that there are exactly 30 complex homotopy types of 6-dimensional nilmanifolds. It is worth remarking that if $\alpha \neq \alpha^{\prime}$, then our nilmanifolds $N_{\alpha}$ and $N_{\alpha^{\prime}}$ have different $\mathbb{C}$-minimal models. Indeed, it can be checked that the proof of Proposition 2 above directly extends to the case when the matrix $\Lambda=\left(\lambda_{j}^{i}\right)_{i, j=1, \ldots, 8}$ defined in (13) belongs to $\mathrm{GL}(8, \mathbb{C})$. In conclusion, our main result in Theorem 1 extends to the complex case, i.e., there are infinitely many complex homotopy types of 8-dimensional nilmanifolds admitting a generalized complex structure of ever type $k$, for $0 \leq k \leq 4$. Now, Corollary 1 is a consequence of the fact that the product nilmanifolds $N_{\alpha} \times \mathbb{T}^{2 m}$ do not admit any Kähler metric.

Author Contributions: Conceptualization, A.L., L.U. and R.V.; writing—original draft preparation, A.L., L.U. and R.V.; writing-review and editing, A.L., L.U. and R.V. All authors have read and agreed to the published version of the manuscript.

Funding: This work has been partially supported by the projects MTM2017-85649-P (AEI/FEDER, UE), E22-17R "Álgebra y Geometría" (Gobierno de Aragón/FEDER), and UZCUD2019-CIE-02 (CUD-Zaragoza, AGM).

Acknowledgments: The authors would like to thank the anonymous referees for their helpful comments and suggestions.

Conflicts of Interest: The authors declare no conflict of interest.

\section{References}

1. Thurston, W.P. Some simple examples of symplectic manifolds. Proc. Am. Math. Soc. 1976, 55, 467-468. [CrossRef]

2. Tralle, A.; Oprea, J. Symplectic Manifolds with No Kähler Structure; Lecture Notes in Mathematics; Springer: Berlin, Germany, 1997, Volume 1661.

3. Hasegawa, K. Minimal models of nilmanifolds. Proc. Am. Math. Soc. 1989, 106, 65-71. [CrossRef]

4. Hitchin, N.J. Generalized Calabi-Yau manifolds. Q. J. Math. 2003, 54, 281-308. [CrossRef]

5. Gualtieri, M. Generalized complex geometry. Ann. Math. 2011, 174, 75-123. [CrossRef]

6. Cavalcanti, G.; Gualtieri, M. Generalized complex structures on nilmanifolds. J. Symplectic Geom. 2004, 2, 393-410. [CrossRef]

7. Angella, D.; Calamai, S.; Kasuya, H. Cohomologies of generalized complex manifolds and nilmanifolds. J. Geom. Anal. 2017, 27, 142-161. [CrossRef]

8. Salamon, S. Complex structures on nilpotent Lie algebras. J. Pure Appl. Algebra 2001, 157, 311-333. [CrossRef] 
9. Bazzoni, G.; Muñoz, V. Classification of minimal algebras over any field up to dimension 6. Trans. Am. Math. Soc. 2012, 364, 1007-1028. [CrossRef]

10. Latorre, A.; Ugarte, L.; Villacampa, R. A family of complex nilmanifolds with infinitely many real homotopy types. Complex Manifolds 2018, 5, 89-102. [CrossRef]

11. Sullivan, D. Infinitesimal Computations in Topology. Inst. Hautes Études Sci. Publ. Math. 1977, 47, $269-331$. [CrossRef]

12. Griffiths, P.; Morgan, J. Rational Homotopy Theory and Differential Forms; Progress in Mathematics, Birkhäuser; Springer: Berlin, Germany, 1981.

13. Deligne, P.; Griffiths, P.; Morgan, J.; Sullivan, D. Real homotopy theory of Kähler manifolds. Invent. Math. 1975, 29, 245-274. [CrossRef]

14. Mal'cev, I.A. A class of homogeneous spaces. Am. Math. Soc. Transl. 1970, 4, 13-20.

15. Nomizu, K. On the cohomology of compact homogeneous spaces of nilpotent Lie groups. Ann. Math. 1954, 59, 531-538. [CrossRef]

16. Félix, Y.; Halperin, S.; Thomas, J.C. Rational Homotopy Theory; Graduate Texts in Mathematics 205; Springer: Berlin, Germany, 2001.

17. Latorre, A.; Ugarte, L.; Villacampa, R. The ascending central series of nilpotent Lie algebras with complex structure. Trans. Am. Math. Soc. 2019, 372, 3867-3903. [CrossRef]

18. Bazzoni, G.; Freibert, M.; Latorre, A.; Meinke, B. Complex symplectic structures on Lie algebras. arXiv 2018, arXiv:1811.05969.

19. Gong, M.-P. Classification of Nilpotent Lie Algebras of Dimension 7 (Over Algebraically Closed Fields and $\mathbb{R}$ ). Ph.D. Thesis, University of Waterloo, Waterloo, ON, Canada, 1998.

(C) 2020 by the authors. Licensee MDPI, Basel, Switzerland. This article is an open access article distributed under the terms and conditions of the Creative Commons Attribution (CC BY) license (http://creativecommons.org/licenses/by/4.0/). 